Few-Body Systems 0, 1-6 (2018)

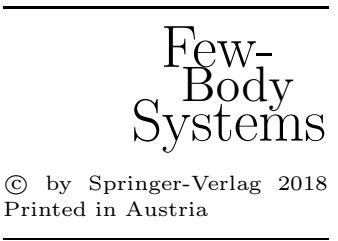

\title{
Correlations in hot and dense quark matter
}

\author{
S. Mattiello ${ }^{a *}$, M. Beyer ${ }^{a * *}$, T. Frederico ${ }^{b}$, H. J. Weber ${ }^{c}$ \\ ${ }^{a}$ Fachbereich Physik, Universität Rostock, D-18051 Rostock, Germany \\ ${ }^{b}$ Dep. de Física, Instituto Tecnológico de Aeronáutica, Centro Técnico Aeroespacial, \\ 12.228-900 São José dos Campos, São Paulo, Brazil \\ ${ }^{c}$ Dept. of Physics, University of Virginia, Charlottesville, VA 22904, U.S.A.
}

\begin{abstract}
We present a relativistic three-body equation to investigate threequark clusters in hot and dense quark matter. To derive such an equation we use the Dyson equation approach. The equation systematically includes the Pauli blocking factors as well as the self energy corrections of quarks. Special relativity is realized through the light front form. Presently we use a zero-range force and investigate the Mott transition.
\end{abstract}

\section{Introduction}

There is increasing interest to investigate nuclear and quark matter under a variety of conditions. Results of lattice simulations suggest a new state of matter, known as quark gluon plasma (see, e.g., Refs. [1]). Recently, this predicted state of matter has been claimed to have been observed by experiments, see e.g. Ref. [2]. Whereas lattice calculations are largely progressing for zero chemical potential $(\mu=0)$ including dynamical quarks, see e.g. [3], calculations at finite $\mu$ are more difficult to implement, see e.g. [4] for treatment with a small finite chemical potential. In view of the present status of lattice calculations it seems inevitable to employ further modeling of QCD if one wants to explore the full phase diagram. Effective theories and models at various stages suggest a rich phase structure at different temperatures and densities, see e.g. [5]. A sketch of the QCD density-temperature plane is shown in Figure 11. Another interesting feature is quark pairing that leads to the possibility of color superconductivity analogous Cooper or nucleon pairing. The phase structure of QCD and in turn the equation of state can be explored using specific signals in heavy ion collision, see e.g. summary in [6]. An understanding of the QCD phases is also important for various astrophysical questions, in particular to explore the structure of neutron stars.

*E-mail address: stefano.mattiello@physik.uni-rostock.de

**E-mail address: michael.beyer@physik.uni-rostock.de 


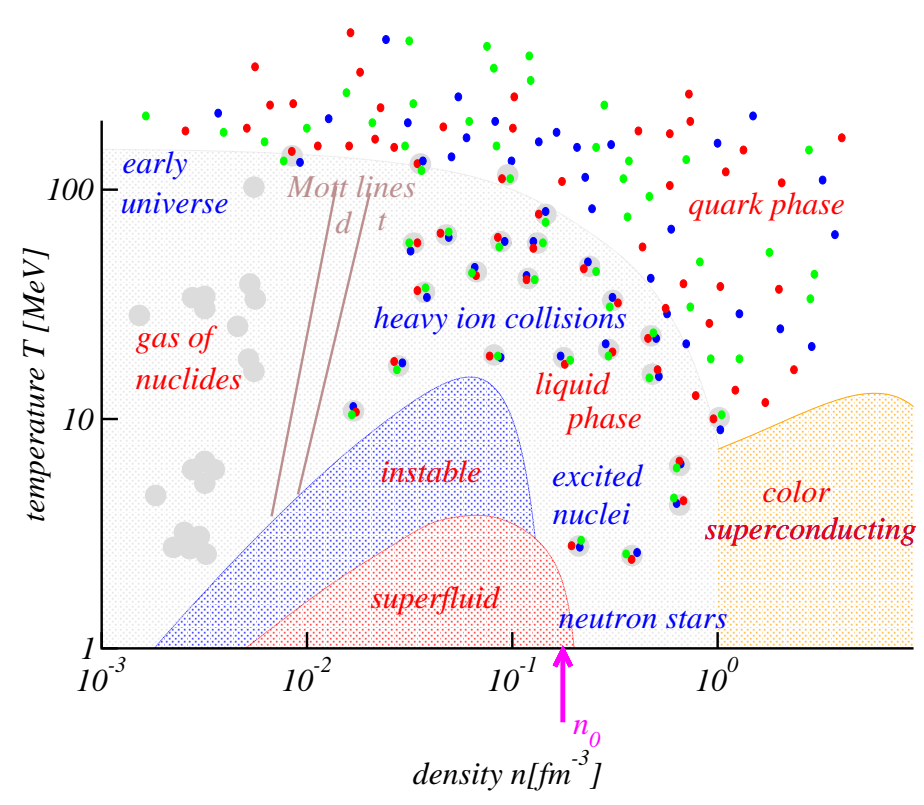

Figure 1. Sketch of the phase diagram of Quantum Chromodynamics.

An aspect that has little been investigated in this context is the appearance of three-quark correlations. We argue that in the vicinity of the phase transition three-quark correlations should play an important role since, below the critical temperature, nucleons (that can be dominantly described as a bound state of three valence quarks) are the relevant degrees of freedom. Three quark correlations might as well influence quark pairing, a question that has recently been addressed for the case $T=0$ [7].

For a systematic investigation of three-quark correlations one needs to derive a suitable relativistic three-body equation that includes aspects of a medium at finite temperatures and densities. In the past years utilizing the Dyson approach we have derived suitable in-medium equations to treat three- and the four-correlations in a medium of finite temperatures and densities, see e.g. [8]. These equations are generalized here to include special relativity. To this end we use the light front approach [9]. Except for details in the treatment of the medium the use of the light front at finite densities is similar to that suggested in Ref. [10].

\section{Theory}

For the time being we consider a zero-range interaction. A simple effective theory of zero range is provided by the Nambu Jona-Lasinio model [11]. Despite well known shortcomings, such as missing confinement, this model reflects basic features of QCD. An overview on the use of the NJL model in the context of finite temperatures and densities can be found e.g. in Ref. [12]. Note that due to screening effects the effective potential between quarks may loose the confining property above the critical temperature 13]. Although the role of confinement needs certainly to be clarified the main focus of our approach is on the structure of the relativistic in-medium equation. 


\subsection{Self energy-correction}

In Hartree-Fock approximation the self-energy correction induced by the medium that changes the constituent mass $m$ is given by the gap equation. For the NJL model the gap equation leads to effective masses $m(\mu, T)$ that depend on the temperature $T$ and the chemical potential $\mu$ of the medium. For $T=10 \mathrm{MeV}$, e.g. $m(100,10)=300, m(200,10)=300, m(300,10)=289, m(307,10)=279$ (units $[\mathrm{MeV}])[14,[15]$. As the chemical potential increases for a given temperature the quark mass becomes smaller; same for a given chemical potential while increasing temperature. To proceed we use the light front formalism. As a Hamiltonian formulation, this approach allows for a relativistic description of the medium in terms of the statistical operator. The Fermi-Dirac distribution function

$$
f\left(k^{+}, \boldsymbol{k}_{\perp}\right)=\left(\exp \left[\frac{1}{k_{B} T}\left(\frac{k_{\mathrm{on}}^{-}+k^{+}}{2}-\mu\right)\right]+1\right)^{-1}
$$

is expressed in terms of light front form momenta that are defined by $\boldsymbol{k}_{\perp}=$ $\left(k_{x}, k_{y}\right)$ and $k^{ \pm}=k_{0} \pm k_{z}$. This treatment coincides with the definition of blocking factors in the limit $T \rightarrow 0$ given in Ref. [10]. Note that the on $k^{-}$-shell light front energy $k_{\text {on }}^{-}=\left(\boldsymbol{k}_{\perp}^{2}+m(\mu, T)^{2}\right) / k^{+}$depends on $\mu$ and $T$.

\subsection{Two-body case}

The technical difficulties including angular momentum in relativistic many-body systems are well known. For the time being we average over the spin projections which means that the spin degrees of freedom are washed out in the medium. This will be improved while the investigation proceeds along the lines suggested in Ref. [16]. Also we expect antiparticle degrees of freedom to be of minor importance for a zero range interaction on the light front [17]. In essence this leads to bose-type relativistic few-body equations including medium effects. The solution for the two-body propagator $\tau\left(M_{2}\right)$ for a zero-range interaction is given by [17]

$$
\tau\left(M_{2}\right)=\left(i \lambda^{-1}-B\left(M_{2}\right)\right)^{-1} .
$$

where the expression for $B\left(M_{2}\right)$ in the rest system of the two-body system is

$$
B\left(M_{2}\right)=-\frac{i}{(2 \pi)^{3}} \int \frac{d x d^{2} k_{\perp}}{x(1-x)} \frac{1-f\left(x, \boldsymbol{k}_{\perp}^{2}\right)-f\left(1-x, \boldsymbol{k}_{\perp}^{2}\right)}{M_{2}^{2}-M_{20}^{2}},
$$

where $M_{20}^{2}=\left(\boldsymbol{k}_{\perp}^{2}+m^{2}\right) / x(1-x)$ and $x=k^{+} / P_{2}^{+}$.

\subsection{Three-body case}

The solution for the two-body propagator $\tau\left(M_{2}\right)$ is the input for the relativistic three-body equation. The inclusion of finite temperature and chemical potential is determined by the Dyson equations as explained, e.g. in Ref. [8]. With the introduction of the vertex function $\Gamma$ the equation becomes

$$
\begin{aligned}
& \Gamma\left(y, \boldsymbol{q}_{\perp}\right)=\frac{i}{(2 \pi)^{3}} \tau\left(M_{2}\right) \int_{M^{2} / M_{3}^{2}}^{1-y} \frac{d x}{x(1-y-x)} \\
& \int^{k_{\perp}^{\max }} d^{2} k_{\perp} \frac{1-f\left(x, \boldsymbol{k}_{\perp}^{2}\right)-f\left(1-x-y,(\boldsymbol{k}+\boldsymbol{q})_{\perp}^{2}\right)}{M_{3}^{2}-M_{03}^{2}} \Gamma\left(x, \boldsymbol{k}_{\perp}\right),
\end{aligned}
$$


where $m=m(\mu, T)$,

$$
k_{\perp}^{\max }=\sqrt{(1-x)\left(x M_{3}^{2}-m^{2}\right)},
$$

and the mass of the virtual three-particle state in the rest system is given by

$$
M_{03}^{2}=\frac{\boldsymbol{k}_{\perp}^{2}+m^{2}}{x}+\frac{\boldsymbol{q}_{\perp}^{2}+m^{2}}{y}+\frac{(\boldsymbol{k}+\boldsymbol{q})_{\perp}^{2}+m^{2}}{1-x-y} .
$$

The blocking factors, $1-f-f$ that appear in (函) can be rewritten as $\bar{f} \bar{f}-f f$, where $\bar{f}=1-f$ to exhibit the particle and the hole blocking. For $T \rightarrow 0, \bar{f}$ coincides with $\theta\left(k-k_{F}\right)$ that cuts the integrals below the Fermi momentum. Note that a blocking factor $\bar{f} \bar{f} \bar{f}$ as suggested e.g. in [0] leads to double blocking of the spectator quark for a two-body kernel at finite temperatures.

\section{Results}

For the time being we assume a bound state $M_{2 B}$ in the two-body subsystem. This can be relaxed while the investigation proceeds and more realistic models are implemented. Our main focus is on effects the medium has on the competition between two and three-quark states. This is particularly relevant in the vicinity of the critical temperatures. A first step towards this aim here is the investigation of the Mott transition from the three-body bound state to the two body $(2+1)$ channel. In passing we note that for the three nucleon system the Mott transition of ${ }^{3} \mathrm{He}$ or ${ }^{3} \mathrm{H}$ is to the three-body channel [18]. To this end, we vary $M_{2 B}$ implicitly choosing a particular model strength $\lambda$ in (2) for each value of $M_{2 B}$. This is shown in Figure 2. For a given $M_{2 B}$ the solid line reflects the corresponding three-body bound state for the isolated system. For a temperature of $T=10 \mathrm{MeV}$ the various

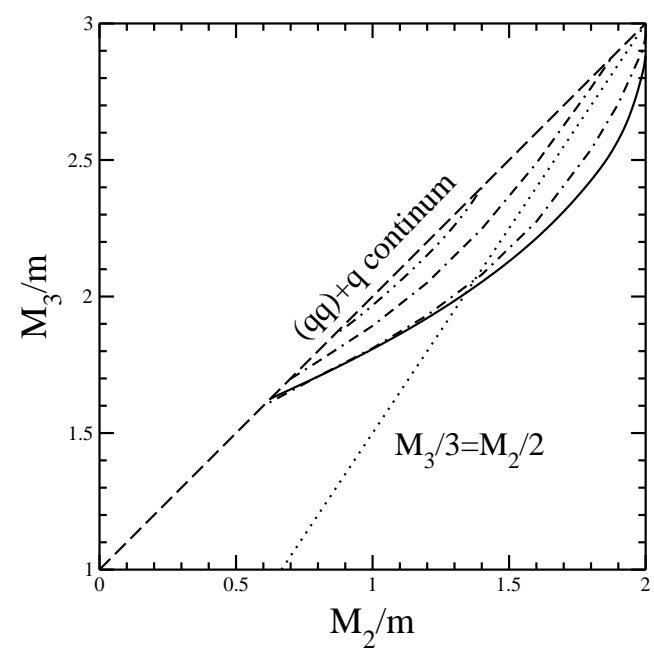

Figure 2. Masses of two-quark and threequark bound states in units of quark mass at $T=10 \mathrm{MeV}$ for different chemical potentials $\mu$ (dashed-dotted line coding as in Figure 3). Further explanations see text.

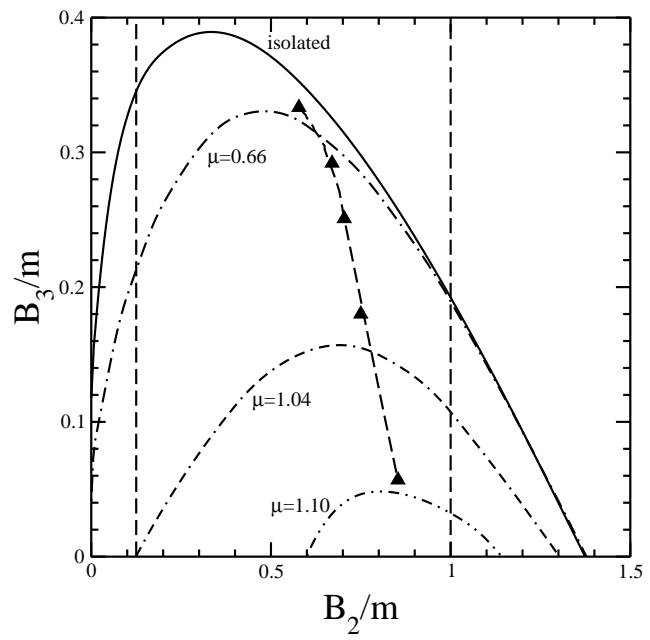

Figure 3. Correlations between two-quark and three-quark binding energies in units of quark mass at $T=10 \mathrm{MeV}$ for different chemical potentials $\mu$, as indicated. Dashed with triangles [7], further explanations see text. 


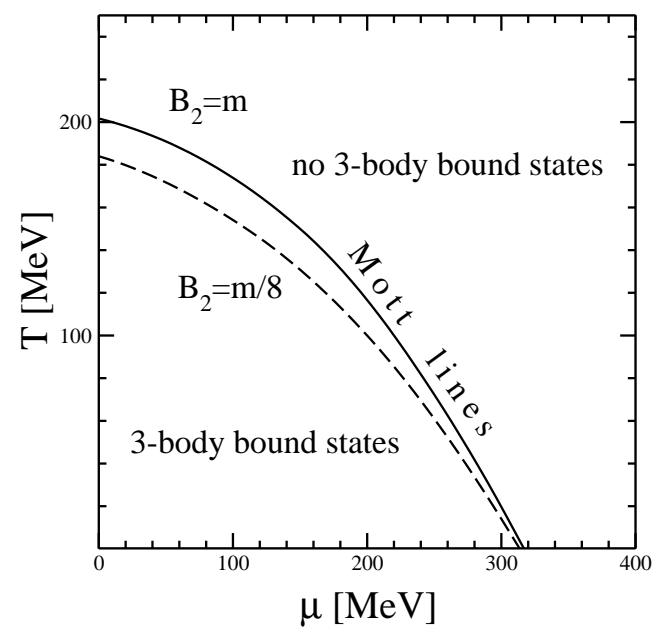

Figure 4. Mott lines for the three-body system at rest in the medium. For values of $T$ and $\mu$ below the Mott lines three-body bound states can be formed. Solid line for $M_{2}=m$, dashed line for $M_{2}=15 \mathrm{~m} / 8$.

dashed-dotted lines correspond to increasing chemical potential (see Figure 3 for the corresponding values of $\mu$ ). The long dashed line is the two-body continuum. In a simple chemical picture of an equilibrium system the dotted line indicates the relative importance for the clusters due to the law of mass action. In Figure 3 we show the binding energies

$$
\begin{aligned}
& B_{3}(\mu, T)=m(\mu, T)+M_{2 B}(\mu, T)-M_{3 B}(\mu, T) \\
& B_{2}(\mu, T)=2 m(\mu, T)-M_{2 B}(\mu, T) .
\end{aligned}
$$

The solid line refers to the isolated case and the various dashed-dotted lines again represent the in-medium results for different chemical potentials at $T=10$ $\mathrm{MeV}$. In addition, we refer to the NJL results (dashed line with triangles) given in Ref. [7] for $m=450 \mathrm{MeV}$ and $T=0$ : The triangles are for values of $\mu(T=$ $0) \simeq 1.0,1.05,1.08,1.12,1.22$ (top to bottom) in units of the respective $m(\mu, 0)$. The dashed vertical lines reflect specific values for $M_{2 B}=m$ and $M_{2 B} \simeq 1.88 m$ in our model covering a wide range of $M_{2 B}$. For a given two-body binding energy and increasing chemical potential we find weaker three-body binding. This leads to the disappearance of the three-quark bound state for a certain value of the chemical potential which is known as Mott transition, $B_{3}\left(\mu_{\text {Mott }}, T_{\text {Mott }}\right)=0$. The values of $T$ and $\mu$ for which this transition occurs is plotted in Fig. 1 for the two different models given above. Clearly the behavior qualitatively reflects the confinement deconfinement phase transition.

\section{Conclusion}

In conclusion, we have given a consistent equation for a relativistic three-quark system in a medium of finite temperature and density. This equation includes the dominant effects of the medium, viz. Pauli blocking and self energy corrections. As many details need to be improved, this first calculation shows that for a large range of models the Mott transition agrees qualitatively with the phase transition expected from other sources. Based on this approach it is now possible to systematically investigate the influence of three-quark correlations on the critical temperature and the onset of color superconductivity at high density. 
Acknowledgement. Work supported by Deutsche Forschungsgemeinschaft.

\section{References}

1. Karsch F.: Nucl. Phys. Proc. Suppl. 83, 14 (2000); Karsch F., Laermann E., and Peikert A.: Nucl. Phys. B 605, 579 (2001); Alford M., Kapustin A. and Wilczek F: Phys. Rev. D 59, 054502 (1999)

2. Heinz U. and Jacob M.: nucl-th/0002042

3. Ejiri S.: Nucl. Phys. Proc. Suppl. 94, 19 (2001) hep-lat/0011006

4. Ejiri S. et al.: hep-lat/0110080

5. Alford M.: hep-ph/0102047

6. Blaizot J. P.: nucl-th/0107025

7. Pepin S., Birse M. C., McGovern J. A. and Walet N. R.: Phys. Rev. C 61, 055209 (2000)

8. Beyer M.: Few-Body Systems, this issue (2001), nucl-th/0110044

9. Dirac P. A.: Rev. Mod. Phys. 21, 392 (1949)

10. Miller G. A. and Machleidt R.: Phys. Rev. C60, 035202 (1999); Miller G. A.: Prog. Part. Nucl. Phys. 45, 83 (2000)

11. Nambu Y. and Jona-Lasinio G.: Phys. Rev. 122, 345-358 (1961); Nambu Y. and Jona-Lasinio G.: Phys. Rev. 124, 246-254 (1961)

12. Klevansky S. P.: Rev. Mod. Phys. 64, 649 (1992)

13. Petreczky P. et al.: hep-lat/0103034

14. Schmidt S. M., diploma thesis, Rostock 1993 and private communication.

15. Beyer M., Mattiello S., Frederico T., Weber H. J.: Phys. Lett. B (2001), in print, hep-th/0106219

16. Beyer M., Kuhrts C. and Weber H. J.: Annals Phys. 269, 129 (1998)

17. Frederico T.: Phys. Lett. B 282, 409 (1992)

18. Beyer M., Schadow W., Kuhrts C. and Röpke G.: Phys. Rev. C 60, 034004 (1999) 